\title{
ReaR
}

\section{¿Disponemos de un protocolo "ideal" en la intubación con fibrobroncoscopio en el paciente con vía aérea difícil anticipada? Revisión}

Artículo original: Cabrini L, Baiardo Redaelli M, Ball L, Filippini M, Fominskiy E, Pintaudi M, et al. Awake Fiberoptic Intubation Protocols in the Operating Room for Anticipated Difficult Airway: A Systematic Review and Meta-analysis of Randomized Controlled Trials. Anesth Analg. 2019 May;128(5):971-980. doi: 10.1213/ANE.0000000000004087 (ubMed)

Muñoz Ávalos N, Palamidessi Domínguez J

H.U. Puerta de Hierro, Madrid.

\section{Resumen}

La intubación con fibrobroncoscopio en el paciente despierto supone una buena opción para aquellos pacientes con vía aérea difícil conocida que se van a someter a una intervención quirúrgica programada, lo cual permite al anestesiólogo responsable idear un plan de acción para evitar un desenlace no deseado. Son muchos los protocolos que se aplican para ello, pero, la mayoría de las veces, obedecen a la experiencia de cada anestesiólogo y a los resultados obtenidos con su aplicación en la práctica asistencial habitual.

Este artículo es la primera revisión sistemática de ensayos clínicos aleatorizados que se centran en este abordaje. Para ello, se seleccionó principalmente información de cuatro áreas de trabajo: premedicación, sedación, anestesia local y uso de dispositivos y técnicas auxiliares que faciliten la intubación en el paciente despierto. La conclusión final a la que llegan los autores de este artículo es la existencia de gran heterogeneidad en lo que a estos aspectos se refiere, sin que ninguno de los protocolos haya demostrado superioridad en seguridad y eficacia.

\section{Introducción}

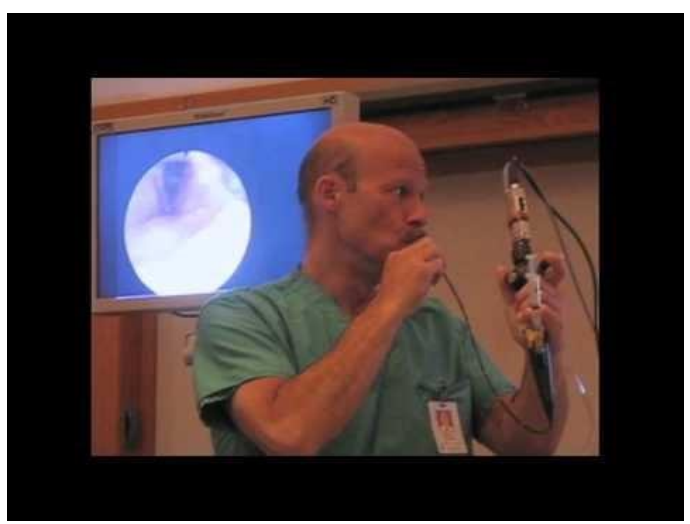

La intubación con fibrobroncoscopio en el paciente despierto supone una buena opción para aquellos pacientes con vía aérea difícil conocida que se van a someter a una intervención quirúrgica programada, lo cual permite al anestesiólogo responsable idear un plan de acción para evitar un desenlace no deseado. Son muchos los protocolos que se aplican para ello, pero, la mayoría de las veces, obedecen a la experiencia de cada anestesiólogo y a los resultados obtenidos con su aplicación en la práctica asistencial habitual.

Este artículo es la primera revisión sistemática de ensayos clínicos aleatorizados que se centran en este abordaje. Para ello, se seleccionó principalmente información de cuatro áreas de trabajo: premedicación, sedación, anestesia local y uso de dispositivos y técnicas auxiliares que faciliten la intubación en el paciente despierto. La conclusión final a la que 
llegan los autores de este artículo es la existencia de gran heterogeneidad en lo que a estos aspectos se refiere, sin que ninguno de los protocolos haya demostrado superioridad en seguridad y eficacia.

La incidencia de vía aérea difícil, definida como aquella situación clínica en la cual un anestesiólogo entrenado experimenta dificultad para la ventilación con mascarilla, la intubación orotraqueal, o ambas, tiene una incidencia descrita muy variable $(0,5-10 \%)$ (1) pero relevante por las consecuencias catastróficas para el paciente si no hacemos un manejo adecuado. A este respecto, una situación que debemos evitar por su potencial morbimortalidad es aquella del paciente "no ventilable-no intubable" que conduce, si no actuamos de una forma adecuada e inmediata, a un compromiso de la oxigenación y secundariamente a un desenlace fatal e incluso la muerte del paciente.

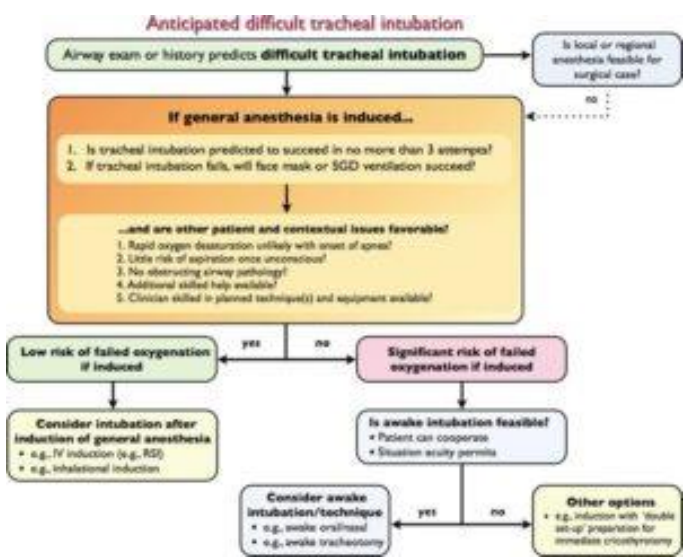

https://anestesiar.org/2014/recomendacionespara-el-manejo-de-la-via-aerea-dificil-2a-partela-via-aerea-anticipada/

Can J Anaesth. 2013 Nov;60(11):1119-38

Un escenario particular es aquel con vía aérea difícil ya conocida en el cual la intubación con el paciente despierto con fibrobroncoscopio es ya considerada "gold standard". En este sentido, es mucha la literatura publicada que avala los beneficios de dicho manejo, pero, sin embargo, no se ha descrito ningún estándar sobre el tipo de premedicación, sedación y protocolo a seguir en este contexto.

\section{Resumen}

Objetivo:

Establecer la relación entre las diferentes técnicas y medidas farmacológicas usadas para la intubación con fibrobroncoscopio en paciente despierto y las consecuencias que estas pueden tener en términos de efectos adversos, muerte $u$ otros eventos.

\section{Métodos:}

Se realiza una revisión sistemática de ensayos clínicos aleatorizados y controlados comparando las diferentes técnicas empleadas mediante búsquedas en PubMed, BioMed Central, Embase y Cochrane.

Se seleccionaron estudios con los siguientes criterios de inclusión: intubación despierto con fibrobroncoscopio en paciente con vía aérea difícil en intervención quirúrgica programada; ensayos controlados aleatorizados comparando distinta premedicación, sedación, anestesia local y uso de dispositivos auxiliares. Se excluyeron: escenarios de intubación diferentes al quirófano, estudios comparativos de intubación despierto con fibrobroncoscopio versus otros dispositivos y técnicas y los estudios basados en simulación.

Los principales outcomes fueron la tasa de éxito, muerte y otros eventos adversos potencialmente fatales. 


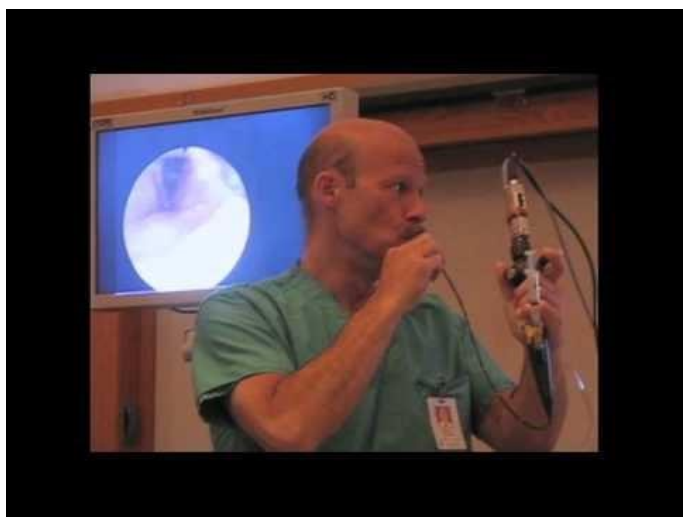

ttps://anestesiar.org/2010/spray-as-yougo-con-lidocaina-al-2-vs-4-para-laintubacion-despierto-con-

fibrobroncoscopio-flexible-delpaciente-con-via-aerea-dificil/

\section{Resultados:}

La búsqueda bibliográfica encontró 2150 artículos de los cuales finalmente se incluyeron en la revisión sistemática 37 ensayos clínicos aleatorizados que incluían un total de 2045 pacientes. En el global de los estudios se encontró una incidencia del $0.59 \%$ de fracaso de intubación y del $0.34 \%$ de eventos adversos graves, de los cuales ninguno de ellos resultó en daño permanente o muerte gracias a su identificación $\mathrm{y}$ tratamiento inmediato.

\section{Conclusión:}

Existe una gran heterogeneidad de estudios en lo que a los aspectos periprocedimiento se refiere, sin hallarse superioridad de ninguno de los protocolos utilizados frente a los demás. No obstante, concluye que son necesarios más estudios que permitan evaluar el impacto de los distintos protocolos en los resultados clínicos del paciente.

\section{Análisis crítico del artículo}

La mayoría de las Sociedades de Anestesiología han desarrollado guías prácticas y algoritmos de toma de decisiones para facilitar la prevención, diagnóstico y tratamiento de los diferentes casos de vía aérea difícil (2).

La selección de la técnica (técnica no invasiva utilizando fibrobroncoscopio, vídeolaringoscopio $\mathrm{o}$ a través de dispositivos supraglóticos, frente a una técnica invasiva como la vía aérea quirúrgica, la ventilación yet o la intubación retrógrada) depende de la cirugía, de la situación del paciente y de la habilidad y preferencias del anestesiólogo (3).

Se estima que la intubación con fibrobroncoscopio en un paciente despierto es exitosa en el $88-100 \%$ de los casos (4) para lo cual se requiere toda una planificación protocolizada en los diferentes pasos del proceso (principalmente, premedicación, sedación, anestesia local) que debe ser evaluada periódicamente para asegurar el cumplimiento de los principales ítems sobre el paciente fundamentalmente en términos de seguridad, eficacia $y$ confortabilidad.

El artículo que nos ocupa analiza los ensayos clínicos aleatorizados publicados en relación con la intubación con fibrobroncoscopio en el paciente despierto con el fin de establecer la superioridad estadística en seguridad y eficacia de unos sobre otros para su recomendación y aplicabilidad en nuestra práctica asistencial.

Para ello tras revisar 37 estudios randomizados controlados llegan a la conclusión de la existencia de un amplio rango de protocolos similares en el cumplimiento de los objetivos antes citados con una incidencia $<1 \%$ de eventos adversos o técnica fallida. Realmente resulta difícil llegar a aseveraciones homogéneas y concluyentes cuando se comparan estudios heterogéneos en tanto en cuanto los protocolos difieren no solo 
en los fármacos empleados (tipo, dosis, técnica de administración) sino también en las técnicas auxiliares complementarias al procedimiento (gafas nasales, anestesia local, gafas de alto flujo, etc ...).

Un factor limitante que los autores de esta revisión ponen de manifiesto es la cifra global pequeña de pacientes estudiados a pesar del importante número de estudios revisados, así como la baja frecuencia en los mismos del empleo de ciertos fármacos como la ketamina y otros dispositivos auxiliares, excluyendo con ello alternativas que, probablemente sean igualmente válidas.

Una premisa que da lugar a pocas dudas es la idoneidad de realizar una intubación con fibrobroncoscopio con el paciente despierto en aquellos casos con vía aérea difícil ya conocida previamente con el objetivo de evitar la potencial morbimortalidad asociada a dificultad o fallo en la ventilación e imposibilidad de intubación (4). Claramente, la dificultad de esto se incrementa sin una efectiva supresión de la náusea y de la tos (5) para lo cual se requiere un adecuado nivel de sedación que no suponga compromiso de la vía aérea ni añada otros riesgos vinculados a la misma. Tanto es así, que la falta de cooperación del paciente, las limitaciones del equipo y la falta de habilidad del operador se encuentran entre los factores que pueden determinar el fallo de una técnica anestésica que precise la colaboración del paciente (3).

Por tanto y partiendo de las premisas antes citadas, el anestesiólogo debe aplicar aquel protocolo que más se ajuste a la experiencia propia, teniendo presentes las ventajas y desventajas que cada fármaco lleva asociado y los medios auxiliares a su alcance.

\section{Conclusión}

El "4th National Audit Project" (NAP4) nos alerta de la necesidad de extremar precauciones en el paciente con vía aérea difícil, anticipada o no, en los distintos escenarios, con el objetivo último de disminuir la morbimortalidad asociada a un mal manejo de estos pacientes.

En este sentido y paralelo a la importante revolución tecnológica en dispositivos de manejo de vía aérea, son muchos los algoritmos de toma de decisiones que nos facilitan este abordaje. No obstante, cada centro debe establecer las pautas protocolizadas ajustadas a los medios disponibles que en cada centro haya, para garantizar una atención segura, eficaz y de calidad para el paciente.

Lo novedoso de esta revisión viene dado por el objetivo planteado por sus autores de encontrar el protocolo "ideal" en un escenario como es la intubación con fibrobroncoscopio en el paciente despierto con vía aérea difícil anticipada que habitualmente genera mucha inquietud en el personal que atiende al paciente $\mathrm{y}$ ansiedad en el mismo.

Si bien es cierto que el resultado del estudio no alcanza la finalidad esperada, también lo es que la conclusión a la que llegan nos aporta un respiro al considerar que todo protocolo en esta situación es bueno siempre y cuando se haga con un adecuado control $y$ conocimiento de las técnicas de premedicación, anestesia local, sedación y uso de medidas auxiliares que sirvan de hilo conductor a una resolución exitosa.

No obstante, todo protocolo debe ser sometido a evaluaciones y auditorías periódicas que garanticen que nuestra pauta se ajusta los estándares requeridos 
para que pueda ser extensiva $y$ reproducida.

\section{Bibliografía}

1. Xu Z, Ma W, Hester DL, Jiang Y. Anticipated and unanticipated difficult airway management. Curr Opin Anaesthesiol. 2018; 31(1): 96-103. (PubMed)

2. Yepes Temiño MJ, Panadero Sánchez A, Callejas González R, Carrascosa Moreno F, Pérez Valdivieso JR. Evaluación de la eficacia y seguridad de un protocolo de intubación mediante fibroscopio en paciente despierto. Estudio descriptivo retrospectivo. Rev Esp Anestesiol Reanim. 2011; 58: 8084. (HTML)

3. Gomez-Ríos MA, Gaitini L, Matter I, Somri M. Guías y algoritmos para el manejo de la vía aérea difícil. Rev Esp
Anestesiol Reanim. 2018; 65(1):41-48. (HTML)

4. Wong J, Lee JS, Gar Ling Wong TG, Iqbal R, Wong P. Fiberoptic intubation in airway management: a review article. Singapore Med J. 2019; 60(3):110-118. (PMC)

5. Lee HM, Sakong J, Jee DL. The comparison of feasibility and safety on fiberoptic guided intubation under conscious sedation with remifentanil and propofol. Korean J Anesthesiol. 2013 Sep; 65(3):215-22. ( $\underline{\text { PMC) }}$

Correspondencia al autor

Natalia Muñoz Ávalos nataliamaval@hotmail.com

FEA Anestesiología y Reanimación. H.U. Puerta de Hierro, Madrid

Aceptado para el blog en diciembre de 2019 